\title{
Manejo clínico inicial de la esclerosis lateral amioatrófica
}

\author{
Initial clinical management of amioatrophic lateral sclerosis
}

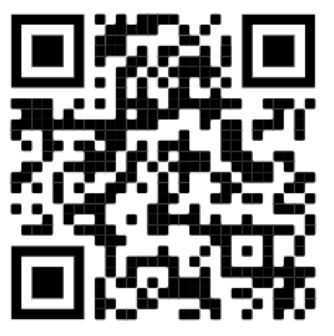

Recibido

\author{
${ }^{1}$ Dr. Ricardo José Charpentier Molina \\ Investigador independiente, San José, Costa Rica \\ (iD https://orcid.org/0000-0003-1095-0723 \\ ${ }^{2}$ Dr. Kevin Vinicio Loaiza Quirós \\ Investigador independiente, San José, Costa Rica \\ (i) https://orcid.org/0000-0003-1076-156X
} \begin{abstract}
$10 / 08 / 2020$

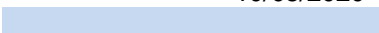

01/10/2020

\section{RESUMEN}

La esclerosis lateral amiotrófica es una enfermedad progresiva que se caracteriza por la degeneración de las neuronas motoras en el cerebro y la médula espinal. Existen dos formas fundamentales de esta enfermedad: la forma esporádica y la forma familiar. Aún no existe claridad sobre su etiología. Las manifestaciones clínicas incluyen signos de daño de las neuronas motoras superiores e inferiores, tanto en las extremidades como en la musculatura bulbar. El diagnóstico continúa siendo fundamentalmente clínico, apoyado por estudios neurofisiológicos. No existe tratamiento curativo. El riluzole es el tratamiento farmacológico de primera línea que ha demostrado retrasar el curso normal de la enfermedad. El edaravone está aprobado por la FDA para el uso en esta enfermedad en conjunto con el riluzole.
\end{abstract}

\begin{abstract}
Corregido $02 / 09 / 2020$
\end{abstract}

Aceptado

PALABRAS CLAVE: esclerosis; neuronas motoras; riluzole; neurología; terapéutica.

\begin{abstract}
Amyotrophic lateral sclerosis is a progressive disease characterized by degeneration of motor neurons in the brain and spinal cord. There are two fundamental forms of this disease: the sporadic form and the familial form. There is still no clarity about its etiology. Clinical manifestations include signs of damage to the upper and lower motor neurons, both in the extremities and in the bulbar musculature. The diagnosis continues to be fundamentally clinical, supported by neurophysiological studies. There is no curative treatment. Riluzole is the first-line drug treatment that has been shown to delay the normal course of the disease. Edaravone is approved by the FDA for use in this disease in conjunction with riluzole.
\end{abstract}

KEYWORDS: esclerosis; neuronas motoras; riluzole; neurología; terapéutica.

\footnotetext{
${ }^{1}$ Médico general, graduado de la Universidad de lberoamerica (UNIBE), cod.MED16745, correo: richarmo96@hotmail.com

2 Médico general, graduado de la Universidad de Costa Rica (UCR), correo: Kevinloaizaquiros@gmail.com
} 


\section{INTRODUCCIÓN}

La esclerosis lateral amiotrófica (ELA) es una patología caracterizada por la degeneración de las neuronas motoras a nivel de sistema nervioso central. Generalmente se manifiesta de forma insidiosa con debilidad focal, que se extiende en el tiempo para involucrar a la mayoría de los músculos, incluyendo el diafragma, lo que puede comprometer la función respiratoria de los pacientes (1).

Las neuronas motoras se dividen en superiores, que se encuentran en la corteza motora a nivel cerebral, e inferiores, que se encuentra en el tronco encefálico y la médula espinal. Las neuronas motoras inferiores (NMI) brindan la inervación muscular (2). Al presentarse una alteración en las neuronas motoras superiores (NMS), clínicamente se evidencia rigidez muscular y espasticidad. Cuando las NMI son las afectadas, inicialmente, presentan irritabilidad eléctrica excesiva, lo que lleva a contracciones musculares espontáneas e involuntarias conocidas como fasciculaciones. A medida que la degeneración neuronal progresa, se pierde conectividad sináptica con sus músculos objetivo, resultando en atrofia muscular (2).

Hasta el momento la ELA una enfermedad sin tratamiento curativo, con una expectativa de vida menor a la población general, y con consecuencias importantes en la vida del paciente que generan necesidad de cuidados permanentes, problemas emocionales y psicológicos (3).

Durante las últimas dos décadas, se ha reconocido que entre el 15 y el $20 \%$ de los pacientes con ELA tienen anormalidades cognitivas progresivas marcadas por cambios de comportamiento, que conducen, en última instancia, a la demencia, conocida como demencia frontotemporal (1).
Es importante conocer las distintas formas de presentación clínica, los estudios necesarios para hacer el diagnóstico y los posibles tratamientos que existen, cuyo objetivo es controlar el curso de la enfermedad.

El objetivo de esta revisión es brindar información sobre el manejo integral inicial de estos pacientes, así como las opciones terapéuticas más nuevas que buscan impactar en la calidad de vida, tanto de los pacientes.

\section{MÉTODO}

Se realizó la búsqueda de fuentes bibliográficas, de las cuales se seleccionaron 18, para la elaboración del presente artículo. Las fuentes de información y consulta se basaron en bases de datos como Scielo, New England Journal of Medicine y JAMA. Se utilizó para la búsqueda palabras claves como "esclerosis lateral amiotrofica" "neurología" y terapéutica".

Se excluyeron artículos con antigüedad de 5 años, artículos de fuentes no confiables. Se incluyeron únicamente artículos y estudios científicos, publicados entre el año 20162020, que incluían el abordaje en el adulto, y tenían como objetivo describir el manejo del paciente con ELA, así como las actualizaciones de los nuevos tratamientos.

\section{EPIDEMIOLOGÍA}

La ELA es la tercera enfermedad neurodegenerativa más frecuente, después de la demencia y la enfermedad de Parkinson.

Junto a sus variantes (esclerosis lateral primaria, atrofia muscular progresiva y parálisis bulbar progresiva), es la 
enfermedad de neurona motora más frecuente en adultos (3).

La enfermedad suele manifestarse entre los 50 y 60 años, aunque existen casos más precoces y también de comienzo posterior. Teniendo en cuenta que la edad media es algo menor que en las otras enfermedades neurodegenerativas, en muchas ocasiones afecta a personas laboralmente activas (3). La esperanza de vida varía según la evolución clínica, sin embargo se estima que después del diagnóstico, tienen una supervivencia de 3 a 5 años, aunque en el $10 \%$ de los casos la supervivencia es superior. Existen dos formas fundamentales de ELA: ELA esporádica y ELA familiar. La ELA familiar supone un $5-10 \%$ de todos los casos. Las manifestaciones clínicas entre ambas son similares (2).

La incidencia es de 1-3 por 100.000 y una prevalencia de 3-5 por 100.000 habitantes. Dado el bajo número de pacientes afectados, se considerada una enfermedad rara (2). Se describe un aumento en el número de casos diagnosticados cada año en el mundo, debido probablemente a una mejora en el diagnóstico y a la mayor supervivencia de la población general (2).

Los factores de riesgo reconocidos son el sexo masculino, la edad avanzada y los antecedentes familiares de ELA, que usualmente se asocian con mutaciones genéticas específicas (4). Muchos factores exógenos se han investigado, incluyendo la exposición a pesticidas, campos electromagnéticos, algunos metales, contaminación por cianobacterias, sin embargo, los factores de riesgo ambiental no han sido concluyentes (4).

Un estudio publicado el 2019 por Marie Ryan y colaboradores, confirmó que hasta el 50\% de la varianza en la ELA tiene una base genética, y que la presencia de la variante C9orf72 es un determinante importante de la heredabilidad. Los familiares de primer grado de individuos con ELA sin una base genética conocida, siguen teniendo un mayor riesgo de desarrollar ELA, en comparación con la población general (5).

\section{PATOGENIA}

La etiopatogenia de la ELA no ha sido claramente establecida a pesar de los distintos estudios a lo largo de los años (6). Actualmente se puede clasificar como ELA familiar (ELAF) y ELA esporádica (ELAE) (7). Aproximadamente un 5-10\% de los casos de ELA son hereditarios (3). El 90\% restante de los casos de ELA son esporádicos (6). En ambos casos se produce un proceso neurodegenerativo que lleva a la muerte de las neuronas motoras.

En la actualidad se considera que esta degeneración es un proceso focal de la NMS $y$ la NMI que avanza continua y separadamente (7). La mayor actividad física, es considerada un factor desencadenante de la enfermedad más que factores de riesgo (3).

\section{CLÍNICA}

La presentación típica de la ELA se caracteriza por la presencia de manifestaciones clínicas de lesión de las NMS y NMI asociado a signos de alteración bulbar y respiratoria (7).

La debilidad muscular es el síntoma más frecuente y se debe a la degeneración progresiva de neuronas motoras. Se manifiesta cuando se ha perdido el $50 \%$ de la de neuronas motoras. Inicialmente afecta a un grupo de músculos y se va difundiendo hacia otros, a medida que avanza la enfermedad (3). Debido al daño motoneurona inferior, la atrofia muscular, 
fasciculaciones, calambres musculares y pérdida de reflejos son manifestaciones comunes de la enfermedad. Como consecuencia la degeneración de la motoneurona superior, se presenta además: espasticidad, hiperreflexia y signos de NMS como el signo de Babinski y además de labilidad emocional, conocida como risa y llanto espasmódicos (2).

Habitualmente los pacientes cursan sin demencia, aunque un $50 \%$ puede presentar alteraciones psicológicas y conductuales. Aproximadamente un $10-15 \%$ presentan signos de demencia frontotemporal $(3,7)$.

Independientemente de que el paciente inicie con manifestaciones de afectación NMS o NMI, el $80 \%$ de los pacientes desarrollarán, signos y síntomas de afectación bulbar, principalmente disfagia, disartria y pérdida de capacidad de la tos, lo que conlleva a mayor riesgo de broncoaspiración, infección respiratoria, neumonía y asfixia, que son las principales causas de muerte en estos pacientes $(2,3)$.

\section{CLASIFICACIÓN DE PATRONES CLÍNICOS}

- La esclerosis lateral amiotrófica clásica: representa del $65 \%$ al $70 \%$ de los casos; generalmente inicia entre el rango de edad entre los 58 y los 63 años (7). Involucra tantos signos de NMS y NMS y usualmente a los 4 años de inicio de la enfermedad, el paciente se encuentra en estado crítico o incluso fallecen. La debilidad muscular es progresiva pero inicialmente es discreta y avanza constantemente con el tiempo. $(2,8)$.

- Esclerosis lateral primaria: representa el $20 \%$ de los casos en el adulto (7). Es síndrome que involucra predominantemente la degeneración de la NMS. Los síntomas inician en los miembros inferiores ascienden de forma relativamente simétrica a los brazos y los músculos bulbares (2,8). Algunos autores sugieren que un criterio diagnóstico de esta variante será el progreso de la enfermedad durante tres años sin signos de NNI; sin embargo, muchos de estos pacientes desarrollan signos de lesión de la NMI, por lo que algunos expertos prefieren denominar este trastorno como "ELA con predominio de daño de $\mathrm{MNS}^{\prime}(7)$.

- Atrofia muscular progresiva: se refiere a un síndrome con afectación predominante de NMI. A diferencia de la ELA clásica, el inicio de la Atrofia muscular progresiva (AMP) puede ocurrir en cualquier región del cuerpo, es más frecuente en hombres y generalmente tiene un inicio posterior (8). Se han documentado casos de supervivencia de 15 años o más. Muchos de estos pacientes presentan signos de daño de la NMS después de muchos años de haber comenzado la enfermedad $(2,7)$.

- Parálisis bulbar progresiva: este patrón comprende del $25 \%$ al $30 \%$ de los casos de ELA y describe a pacientes que manifiestan clínica a nivel de los músculos del habla, la masticación y la deglución, asociado a afectación de la MNI (llamada forma bulbar) y MNS (llamada forma pseudobulbar (8). El inicio bulbar es más frecuente en las mujeres y está altamente asociado con afectación cognitiva y emocional correlacionada directamente con la depresión (8). 
Los pacientes presentan disartria, disfagia, con atrofia y fasciculaciones linguales $(2,7)$. Tempranamente, puede haber presencia de debilidad espástica del maxilar inferior y cierre involuntario de la mandíbula por espasmo; el tiempo de evolución y supervivencia es aproximadamente de uno a dos años (7).

\section{ESTUDIOS DIAGNÓSTICOS}

A todos los pacientes se les deben hacer los siguientes estudios diagnósticos:

- Neurofisiología: la electromiografía con neuroconducciones es una herramienta útil para apoyar el diagnóstico y seguimiento de pacientes con enfermedades de las neuronas motoras, sin embargo los hallazgos deben ser correlacionados con la clínica ya que no son específicos para ELA $(7,9)$. Por otro lado, la velocidad de conducción nerviosa es importante para descartar otras enfermedades $(2,9)$.

- Laboratorio: los estudios de laboratorio contribuyen a descartar otros trastornos, comorbilidades y complicaciones de la enfermedad $(2,9)$. Se deben de solicitar los siguientes estudios: hemograma, reactantes de fase aguda, pruebas de función renal, hepática y tiroidea, electrólitos, electroforesis de proteínas y perfil glucémico (7).

- Neuroimágenes: el principal papel de los estudios de imagen es descartar otras causas de un síndrome piramidal, como tumores, radiculopatías, enfermedad cerebrovascular, mielopatía, etc $(7,9)$. No hay hallazgos específicos en estos pacientes y los estudios son, generalmente, normales. Sin embargo, a medida que la enfermedad progresa, la resonancia magnética puede mostrar atrofia cortical de predominio frontotemporal, y en el segmento anterior de la médula espinal $(2,7,9)$.

- Estudios genéticos: las pruebas genéticas no se solicitan, por rutina, en pacientes con ELA, pero si se sospecha de ELAF o de enfermedad de inicio juvenil, deben solicitarse $(2,7,8)$.

- Biomarcadores de disfunción de motoneurona superior: los biomarcadores objetivos de lesión de NMS pueden ser críticos para el diagnóstico de ELA, ya que los posibles trastornos que imitan, como la neuropatía motora multifocal, la enfermedad de Kennedy y la atrofia muscular espinal de inicio en adultos, pueden presentarse como síndromes puros de NMI $(7,10)$.

- Biomarcadores de disfunción de motoneurona inferior: la evaluación objetiva de la disfunción de la NMI, utilizando técnicas neurofisiológicas, parece ser más sensible que las evaluaciones clínicas. Los estudios de conducción nerviosa, que miden la amplitud del potencial de acción muscular compuesta (CMAP), pueden ser relativamente insensibles para evaluar la degeneración de NMI, debido al proceso de reinervación $(7,10)$.

\section{CRITERIOS DIAGNÓSTICOS}

Para realizar el diagnóstico de ELA, es necesario determinar la presencia de 
enfermedad de la NMS, evidenciada clínicamente, y de la NMI, demostrada clínica o electromiográficamente $(2,3)$. Además se clasifican los pacientes de acuerdo con el número de regiones corporales afectadas de un total de cuatro: bulbar, cervical, torácica y lumbar (conocidos como los CEER) (7).

Dichos criterios demostraron tener baja sensibilidad, e incluso se sabe que muchos pacientes mueren por esta enfermedad sin llegar a cumplirlos; por tal razón, los CEER fueron modificados en el 2008 con el algoritmo de Awaji-Shima, en el que se hicieron algunos cambios, pero se mantuvieron los principios de los criterios CEER. En dicho algoritmo se clasificó la certeza diagnóstica en tres categorías: clínicamente posible, clínicamente probable, clínicamente definida; los criterios de AwajiShima tienen mayor sensibilidad (81\% versus $62 \%$ ), e igual especificidad (95\%) que los CEER (7). Es importante conocer el ALS Funtional Rating Scale (ALSFRS), que establece la capacidad funcional de este tipo de pacientes; de esta forma se evalúan los distintos tratamientos en estudio y su eficacia, además de orientar el manejo del paciente $(2,3)$.

\section{TRATAMIENTO}

Como previamente se ha descrito, esta patología impacta de forma negativa el estilo de vida del enfermo y de las personas con las que convive, por lo que es importante realizar un adecuado abordaje, tanto farmacológico como no farmacológico, para intentar disminuir esta afectación que, en algún punto del curso de la enfermedad, llegará a ser incapacitante, hasta no disponer de tratamientos que modifiquen el curso de la enfermedad.

\section{Abordaje no farmacológico}

- Terapia física: asociada a equipamientos especiales, pueden aumentar la independencia del durante el curso de la ELA. El ejercicio aeróbico suave y de bajo impacto, como caminar, nadar y montar bicicleta estacionaria, pueden fortalecer los músculos no afectados, mejorar la salud cardiovascular, así como combatir la fatiga y la depresión (2,9). Los dispositivos como rampas, aparatos ortopédicos, caminadores y sillas de ruedas, pueden contribuir a conservar la energía y a la movilidad de los pacientes en fases avanzadas (3).

- Terapia de lenguaje: los pacientes con ELA que desarrollan disartria importante, pueden beneficiarse de trabajar con un terapeuta del lenguaje, quien les puede enseñar estrategias para hablar más fuerte y más claro, y de esta manera, le a mantener la habilidad para comunicarse $(3,7)$. También pueden recomendarse sintetizadores de lenguaje computarizado, que usan tecnología de rastreo ocular y así desarrollar formas de responder, sí o no, con los ojos o por otros medios no verbales (9).

- Soporte nutricional: es esencial, pues se ha demostrado que estos pacientes se debilitan si pierden peso $(3,7)$. La educación nutricional dirigida hacia el paciente y sus familiares, facilita la alimentación para se les pueda proporcionar suficientes calorías, fibra y líquidos, así como evitar alimentos que sean difíciles de tragar (9). Se pueden empezar a usar dispositivos de succión 
para retirar el exceso de líquidos o saliva y prevenir la asfixia (9). Cuando ya no puede obtener suficiente nutrición a través de la vía oral, se debe valorar la necesidad una gastrostomía percutánea (3).

- Apoyo para respirar: a medida que los músculos de la respiración son afectados, los pacientes pueden percibir un cansancio durante la actividad física y dificultad respiratoria al dormir o cuando están acostados. Los médicos pueden evaluar la respiración del paciente para determinar cuándo recomendar ventilación no invasiva ( $\mathrm{VNI})(2,3)$. Al inicio puedria ser necesaria solo durante la noche, pero conforme progresa la enfermedad, las concentraciones de oxígeno y dióxido de carbono se ven alteradas, por lo que es recomendable utilizarla todo el tiempo. La VNI mejora la calidad de vida y prolonga la supervivencia de muchas personas con $\operatorname{ELA}(7,9)$. A medida que la enfermedad progresa y los músculos respiratorios se debilitan más, puede ser necesario considerar formas de ventilación mecánica o inclusive una traqueostomía que se conecta a un ventilador $(3,7)$.

\section{Tratamiento farmacológico}

- Riluzole: es el único fármaco que ha demostrado tener un impacto en la ssobrevida en casos de ELA y es el fármaco de primera línea (2). La evidencia de que el riluzole es beneficioso proviene de dos ensayos aleatorizados multicéntricos; en un estudio prospectivo, doble ciego, controlado con placebo en 155 pacientes ambulatorios con ELA, la supervivencia a los 12 meses fue significativamente mayor para los pacientes que recibieron riluzole (100 mg / día) en comparación con los que recibieron placebo (74\% vs $58 \%$ ). Para el subgrupo de pacientes con ELA de inicio bulbar, surgió una ventaja aún mayor para la supervivencia a los 12 meses para el grupo de riluzole $(73 \%$ vs $35 \%$ ) (11).

Un estudio randomizado, doble ciego, realizado por Albert Ludolph et al. sugiere que el uso Rasagiline asociado a Riluzole es una combinación segura, pero no aumenta la sobrevida. En análisis posteriores se sugiere que esta combinación podría modificar la progresión en pacientes con ELA que tenían un ALSFRS de 0,5 o menor, pero se necesitan otros estudios para comprobarlo (12).

- Edaravone: es un fármaco intravenoso para el tratamiento de la ELA, aprobado por la FDA en mayo de 2017. Un estudio aleatorizado con 139 pacientes con ELA, que duró solo seis meses y con criterios algo estrechos de selección, arrojó resultados notables. Se evidenció enlentecimiento en la progresión de la enfermedad en el 33\% de los casos, según el puntaje de la escala ALSFRS-R (2).

Las mediciones de la calidad de vida concordaron, al mostrar que la disminución de fue significativamente más lenta. Las mediciones respiratorias también mejoraron, pero sin alcanzar significación estadística (13).

El edaravone se administra por vía intravenosa en ciclos diarios durante dos semanas, alternando con dos semanas sin tratamiento (2). La semivida del fármaco es de aproximadamente 4,5-6,0 horas, lo que sugiere que la 
administración más frecuente podría tener algún beneficio, sin embargo se necesitan más estudios al respecto. La mayoría de los pacientes en los estudios en los estudios mencionados utilizaban riluzole como tratamiento de base por lo que el uso de ambos fármacos se puede utilizar (13).

\section{Otros fármacos}

El inhibidor de la tirosina cinasa masitinib, es un fármaco que se encuentra en estudio para su utilización en ELA, se han descrito efectos similares a los de riluzole 0 edaravone. Actualmente el mastinib se encuentra en estudios de fase 3 para evaluar su seguridad y eficacia (14).

Asimismo, el descubrimiento de expansiones repetidas de C9orf72 como causa de ELA, reveló una estrategia terapéutica para esa forma más frecuente de la enfermedad. Se cree que muchos de los efectos producidos por esta mutación se deben a la acumulación de ARN transcritos por C9 o f72 (5).

Estos ARN transcritos interactúan en forma aberrante y sirven de matrices para la síntesis de proteínas, muchas de las cuales son tóxicas. Por esta razón se están desarrollando posibles tratamientos, como los oligonucleotidos antisentido que actúan sobre estos ARN transcritos y los degradan (13).

\section{ENFOQUE DE CÉLULAS MADRE}

Durante los últimos años ha habido mucho interés en los tratamientos con células madre para la ELA. Los tratamientos con células madre se encuentran ahora en estudios clínicos de fase 1, 2 y hasta fase 3 (13).
El objetivo de los enfoques actuales es principalmente proteger las neuronas motoras supervivientes y no se busca reemplazar las neuronas motoras muertas (13).

Las células madre tienen el potencial de diferenciarse en células de soporte como los astrocitos, oligodendrocitos o microglia, que pueden brindar un beneficio al disminuir la degeneración de neuronas motoras, producir factores de crecimiento y citosinas antiinflamatorias, así como proporcionar nutrientes y amortiguar el exceso de glutamato (15).

Al ser una fuente autóloga de células madre, los pacientes no requieren tratamiento con inmunosupresores. Las vías de administración usuales son intratecales o intramusculares. La mayoría de los ensayos actuales son pequeños y dirigidos principalmente a evaluar la seguridad. Recientemente se inició, un estudio de fase 3 de células madre, derivadas de médula ósea, inducidas por factor de crecimiento neurotrófico, y se evaluará su eficacia, utilizando la ALSFRS (15).

\section{$\underline{\text { Terapias basadas en células }}$}

- Terapias basadas en astrocitos: en los trabajos publicados recientemente, se describió un original enfoque terapéutico, basado en el uso de astrocitos derivados de células madre embrionarias humanas. Sus hallazgos sugirieron que el suministro intratecal de astrocitos jóvenes y saludables, contribuye asuperar la función neurotóxica de los astrocitos endógenos en la ELA. Es de destacar que los resultados provisionales mostraron una disminución significativa en la progresión de la enfermedad, y no se informaron eventos 
adversos graves relacionados con el tratamiento $(13,15)$.

En este 2020, se iniciará un estudio abierto de fase 1/2a para investigar la seguridad del trasplante de células progenitoras restringidas a la glial humana (hGRPs; Q-Cells®) en la médula espinal lumbar / cervical de pacientes con ELA. Este estudio tiene como objetivo obtener datos preliminares sobre la seguridad, la tolerabilidad y la eficacia temprana del trasplante de QCells® en pacientes con ELA (16).

\section{PRONÓSTICO}

Se ha demostrado en un estudio publicado 2019, que la expansión repetida de c9orf72 confiere una desventaja de supervivencia en la ELA (17). Los antecedentes familiares, el sexo 0 la presencia de variantes de secuencias patógenas adicionales, no se asociaron significativamente con la supervivencia (17).

Un estudio publicado por Rosenbohm, A. en el 2018, sugiere que la proteína de unión al retinol 4 (RBP4) esta inversamente relacionada con el riesgo y el pronóstico de la ELA, lo que sugiere que el metabolismo de la vitamina $A$ o la señalización de insulina podrían estar alteradas en estos pacientes (18). Se necesita más investigación, para aclarar el papel de la resistencia a la insulina en la patogénesis de la ELA (18).

\section{CONCLUSIONES}

La esclerosis lateral amiotrófica es una enfermedad neurodegenerativa que tiene consecuencias devastadoras para el paciente y su familia. Aún no existe claridad sobre la etiología. Las manifestaciones clínicas incluyen signos de daño de las neuronas motoras superiores e inferiores tanto en las extremidades, como en la musculatura bulbar, y en algunos pacientes hay deterioro cognitivo frontotemporal. La ELA clásica es el patrón más frecuente de la enfermedad, sin embargo se deben de conocer los demás patrones clínicos ya que tienen características diferentes especialmente en cuanto a su sobrevida y evolución de la enfermedad. El diagnóstico continúa siendo fundamentalmente clínico, evidenciado por signos y síntomas de afectación en las motoneuronas, apoyado por estudios neurofisiológicos; de estos, la electromiografía ha sido el más útil para el diagnóstico temprano. Los pacientes con ELA se benefician de un abordaje integral y multidisciplinario por lo que la terapia física, la terapia respiratoria y la nutrición son esenciales para mejorar la calidad de vida de estos pacientes. Hasta el momento no existe tratamiento curativo y hasta hace poco, el riluzole era el único fármaco que ha demostrado efectividad para retrasar el uso de ventilación mecánica y prolongar levemente la supervivencia. Recientemente se cuenta también con el edaravone que ha sido aprobado por la FDA para su uso en esta enfermedad en conjunto con el riluzole. Además el inhibidor de la tirosina cinasa masitinib aparentemente podría tener efectos similares al riluzole, pero faltan estudios para aprobar su uso. Se encuentran en estudio terapias prometedoras como el uso de células madres autólogas; sin embargo no hay estudios suficientes para recomendar su adecuada utilización. Se ha demostrado que la expansión repetida de c9orf72 confiere una desventaja de supervivencia en la ELA, debido a que esto genera producción que ARN aberrante que resulta en proteínas neurotóxicas, por esa razón se han planteado tratamientos nuevos con oligonucleotidos antisentido que actúan 
sobre estos ARN transcritos y los degradan, de esta forma se podría tratar esta forma frecuente de la enfermedad. El trasplante de Q-Cells ${ }^{\circledR}$ para el tratamiento en pacientes con ELA se encuentra actualmente en estudios para verificar su seguridad y eficacia, el objetivo de esta terapia a base de células madre diferenciadas, busca evitar o disminuir la toxicidad neuronal producida en la ELA. A pesar de los esfuerzos científicos, no se ha logrado detener el curso de la enfermedad.

\section{REFERENCIAS}

1. Brown R, Al-Chalabi A. Amyotrophic Lateral Sclerosis. NEJM. 2017;(N Engl J Med 2017; 377):162-172. https://doi.org/10.1056/NEJMra1603471

2. Jameson J, Kasper D, Fauci A, Hauser S, Longo D, Loscalzo J et al. Harrison's principles of internal medicine, 20e. 20th ed. Mc Graw Hill Education; 2018; 7639-7656.

3. Perez S, et al. Abordaje de la esclerosis lateral amiotrófica [Internet]. [Consultado 24 Abril 2020]. Madrid, España: Ministerio de sanidad, servicios sociales e igualdad. 2018. Disponible en: https://adelaweb.org/wpcontent/uploads/2017/11/Asociacion-adEla Plan-Nacional-de-ELA.pdf

4. Filippini T, Fiore M, Tesauro M, Malagoli C. Clinical and Lifestyle Factors and Risk of Amyotrophic Lateral Sclerosis [Internet]. [Consultado 25 Abril 2020]. Res. Public Health 2020, $2020 . \quad$ Disponible en:https://res.mdpi.com/d attachment/ijerph/ijerph-17-00857/article deploy/ijerph-17-00857-v2.pdf

5. Ryan M, Heverin M, McLaughlin R, et al. Lifetime Risk and Heritability of Amyotrophic Lateral Sclerosis. JAMANeurology. 2020;76(11):1367-1374. https://doi.org/10.1001/jamaneurol.2019.2044

6. Massachusetts Medical Society. Amyotrophic Lateral Sclerosis. [Internet]. [Consultado 26 Abril 2020]. NEJM Resident 360.org. 2017. Disponible en: https://resident360.nejm.org/clinical-pearls/amyotrophic-lateral-sclerosis$\underline{2}$

7. Zapata-Zapata C, Franco-Dáger E, Solano-Atehortúa J, Ahunca-Velásquez L. Esclerosis lateral amiotrófica: actualización. SCIELO. 2016;vol.29 no.2. https://doi.org/10.17533/udea.iatreia.v29n2a08

8. Grad L, Rouleau G, Ravits J, Cashman N. Clinical Spectrum of Amyotrophic Lateral Sclerosis (ALS). Cold Spring Harbor Perspectives in Medicine. 2016;7(8):a024117. https://doi.org/10.1101/cshperspect.a024117

9. National Institute of Neurological Disorders and Stroke. Esclerosis lateral amiotrófica. [Internet]. [Consultado 26 abril 2020]. National Institute of Neurological Disorders and Stroke (NINDS). 2018. Disponible en: https://espanol.ninds.nih.gov/trastornos/esclerosis lateral amiotrofica.htm

10. Huynh W, Dharmadasa T, Vucic S, Kiernan M. Functional Biomarkers for Amyotrophic Lateral Sclerosis. Frontiers in neurology. 2019. https://doi.org/10.3389/fneur.2018.01141

11. Gálvez N, Goya N, Cudkowicz, M. Disease-modifying treatment of amyotrophic lateral sclerosis. [Internet]. [Consultado 26 abril 2020]. UpToDate [Internet]. 2020. Disponible en: https://www.uptodate.com/contents/disease-modifying-treatment-of-amyotrophic-lateral-sclerosis/print

12. Statland J, Moore D, Wang Y, Walsh M, Mozaffar T, Elman L et al. Rasagiline for amyotrophic lateral sclerosis: A randomized, controlled trial. Pudmed Muscle \& Nerve. 2018;59(2):201-207. https://doi.org/10.1002/mus.26335 
13. Ferreira R. Esclerosis lateral amiotrófica [Internet]. [Consultado 27 abril 2020]. Intramed. 2019;1. Disponible en: https://www.intramed.net/contenidover.asp?contenidoid=92892

14. Martins I. Phase 3 Trial of Masitinib as Add-on ALS Treatment Cleared by FDA [Internet]. [consultado 30 Abril 2020]. Disponible ALS News Today. 2020 en: https://alsnewstoday.com/2020/04/03/fda-clears-ab-science-toopen-phase-3-trial-of-masitinib-add-on-als-treatment/

15. Abdul Wahid S, Law Z, Ismail N, Lai N. Cell-based therapies for amyotrophic lateral sclerosis/motor neuron disease. Cochrane Database of Systematic Reviews 2019, Issue 12. Art. No.: CD011742. https://doi.org/10.1002/14651858.CD011742.pub3

16. Filipi, T, Hermanova Z, Tureckova J, Vanatko, , Anderova, M. Glial Cells-The Strategic Targets in Amyotrophic Lateral Sclerosis Treatment. Journal of Clinical Medicine, 2020; 9(1), p.261. https://doi.org/10.3390/jcm9010261

17. Glasmacher, S., Wong, C., Pearson, I. and Pal, S. Survival and Prognostic Factors in C9orf72 Repeat Expansion Carriers. JAMA Neurology, 2020; 77(3), p.367. https://doi.org/10.1001/jamaneurol.2019.3924

18. Rosenbohm, A., Nagel, G., Peter, R., Brehme, T., Koenig, W., Dupuis, L., Rothenbacher, D. and Ludolph. Association of Serum Retinol-Binding Protein 4 Concentration With Risk for and Prognosis of Amyotrophic Lateral Sclerosis. JAMA Neurology, 75(5), p.600. https://doi.org/10.1001/jamaneurol.2017.5129 